\title{
IV. Biochemistry
}

\section{Tailoring DNA condensates using a novel dicationic lipid with different spacers}

\author{
A.S. Elkady ${ }^{\mathrm{a}}$, Y. Sebyakin ${ }^{\mathrm{b}}$, R.I. Zhdanov ${ }^{\mathrm{c}}$ and A.R. Khokhlov ${ }^{\mathrm{c}}$ \\ ${ }^{a}$ Physics Department, Moscow State University, Moscow 119992, Russia \\ ${ }^{\mathrm{b}}$ M.V. Lomonosov Academy of Fine Chemical Technology, Moscow, Russia \\ ${ }^{\mathrm{c}}$ Institute of Biomedical Chemistry, RAS, 10 Pogodinskaya st., Moscow 119992, Russia \\ Fax: +7095 9328820; E-mail: ashraf_elkady@hotmail.com
}

Introduction: Molecular self-assembly presents a 'bottom-up' approach to the fabrication of objects specified with nanometre precision. DNA molecular structures and intermolecular interactions are particularly amenable to the design and synthesis of complex molecular objects [1]. Particularly, the self assembly of amphiphilic molecules constitute one of the most fundamental mechanisms for the construction of soft condensed matter biomaterials [2]. It is, however, well known that lipids, as well as mixtures of anionic and cationic single chain surfactants, can readily form bilayers [3,4] that can adopt a variety of distinct geometric forms: they can fold into soft vesicles or random bilayers (the so-called sponge phase) or form ordered stacks of flat or undulating membranes [5].

We report on a novel approach for controlling the size and morphology of DNA condensates, using a novel synthesized dicationic lipid (DCL), with several different analogues, varying in their spacer lengths.

Methods: Cationic liposomes were prepared from the DCL $\left[\mathrm{CH}_{3}\left(\mathrm{CH}_{2}\right)_{16} \mathrm{OOC}_{2} \mathrm{Glu}\left(\mathrm{CH}_{3}\right)_{2} \mathrm{~N}^{+}-\right.$ $\left(\mathrm{CH}_{2}\right)_{4}-\mathrm{N}^{+}\left(\mathrm{CH}_{3}\right)_{2} \mathrm{Glu}\left[\mathrm{COO}\left(\mathrm{CH}_{2}\right)_{16} \mathrm{CH}_{3}\right]_{2}$, I, using lipid hydration method for different lipid derivatives. The complexes were prepared by quick equivolumetric mixing of the pDNA (pEGFP-N1, $4.7 \mathrm{kbp}$ ) solution and $\mathrm{CL}$ at at different charge ratios. Scanning Force Microscopy (SFM) was done using NanoScope III-a (Digital Instruments, Santa Barbara, CA) as described elsewhere [6].

Results and discussions: We observed by SFM, DNA condensation induced by spontaneous self assembly with cationic lipid membranes. It is shown (see Fig. 1) that the nature of condensed particles is strongly dependent upon the individual DCL. While liposomes from DCL with short spacers tend to form condensed units of globular and toroidal form $(50-120 \mathrm{~nm})$; unfolded amorphous particles with micrometer sizes were observed for long spacer DCL's. The formation of rods and nanodiscs has been observed for lipids with medium spacer lenghts. In the latter case, the condensed particles were found to stabilize through forming vertices around their outer edges, which frequently have pores. These topological defects were hetherto observed for another Catanionic systems where nanodiscs, in an excess of cationic component, are formed by rejection of excess charges towards the edges [7]. Besides, in such defect-stabilized structure the only other topology to evacuate excess charges is to segregate them in pores [8]. Moreover, producing pores in the bilayer is a way for the system to self-assemble by minimising its curvature energy, since negative gaussian curvature is favoured at high surface charge [9-11].

Conclusions: In the present study we have demonstrated that DNA condensates with controlled size and morphology can be obtained, using a dicationic lipid with different spacer lengths between the two polar heads of lipid chain groups. The structural and topological transformations are mediated by the 

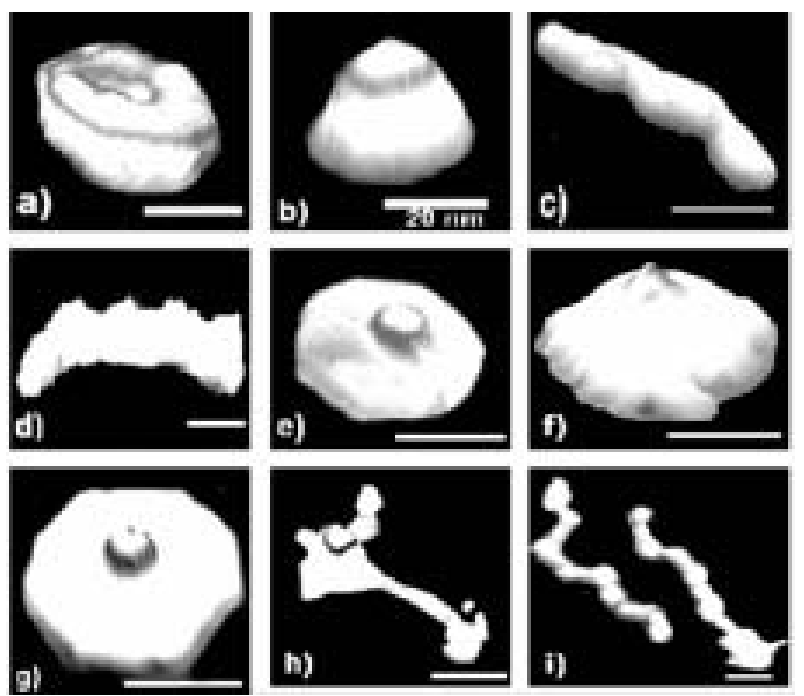

Fig. 1. AFM images for DNA condensates. Shown are different morphologies obtained using a dicationic lipid with different spacer lengths (m) and $\mathrm{L}^{+} / \mathrm{D}^{-}$mass ratios (r). Toroid $m=4, r=6$ (a); globule $m=3, r=10$ (b); rods $m=7, r=6$ (c), (d); nanodiscs $m=7, r=6$ in salt free solution (e), (f), (g); "spaghetti-like" and "beads-on-strings" structures $m=4$, $r=2.1$ respectively (h), (i). The scale bars represent $100 \mathrm{~nm}$ unless mentioned elsewhere.

individual characterestics of the lipid membrane. The differences in lipid membrane regidity, surface charge, and degree of hydrophobicity work cooperatively, leading to DNA condensates in different conformational states. Finally, it's worth mentioning that this study could be rationalized to another cationic surfactants which could be synthesized with different analogues.

\section{References}

[1] E. Winfree et al., Nature 394 (1998), 539-544.

[2] C.L. Gerard Wong et al., Science 288 (2000), 2035-2039.

[3] E. Sackmann and R. Lipowsky, Handbook of Biological Physics 1B (A.J. Hoff, ed.), North-Holland, Amsterdam, 1995.

[4] E.W. Kaler and A.K. Murthy et al., Science 245 (1989), 1371-1374.

[5] M. Dubois and T. Zemb, Curr. Opin. Colloid Interf. Sci. 5 (1997), 27-37.

[6] A. Elkady, Y. Sebyakin, M. Gallyamov, A. Moskovtsev, G. Bischoff, R. Zhdanov and A. Khokhlov, in: Micro- and Nanostructures of Biological Systems, G. Bischoff and H.-J. Hien, eds, Shaker Verlag, Aachen, 2003, in press.

[7] T. Zemb, M. Dubois, B. Demé and T. Gulik-Krzywicki, Science 283 (1999), 816-820.

[8] S.T. Hyde, Colloids Surf. A129-130 (1997), 207-225.

[9] D.R. Nelson and F. Spaepen, Polytetrahedral order in condensed matter, Solid State Phys. 42 (1989), 1-90.

[10] M. Dubois, L. Belloni, T. Zemb, B. Demé and T. Gulik-Krzywicki, Prog. Colloid Polym. Sci. 115 (2000), $238-242$.

[11] M. Dubois and B. Demé et al., Nature 411 (2001), 672-675. 
On the structural-activity relationship for lipoplexes prepared from a newly synthesized dicationic lipid and plasmid DNA

\author{
Ashraf S. Elkady ${ }^{\mathrm{a}, *}$, A. Moskovetsov ${ }^{\mathrm{b}}$, A.R. Jdanov ${ }^{\mathrm{b}}$ and A.R. Khokhlov ${ }^{\mathrm{a}}$ \\ ${ }^{a}$ Physics Department, Moscow State University, Moscow 119992, Russia \\ ${ }^{\mathrm{b}}$ The Institute of General Pathology and Pathological Physiology, 125315 Moscow, Russia
}

Introduction: Recently, there is an increasing interest in the DNA-cationic lipid macromolecular complexes as a non-viral delivery system for gene therapy purposes [1,2]. Yet, detailed studies concerning the molecular mechanisms, causing aggregate formation and its interaction with cells are still lacking. Using atomic force microscopy (AFM), one can elucidate the structural peculiarities for the supramolecular DNA-CL complexes, and identify some of the key parameters that are crucial for optimising gene transfer [3]. In the present study, we emphasize on the structure-activity relationship of lipoplexes prepared from a newly synthesized dicationic lipid, and characterized using AFM. The results obtained highlight some of the structural and morphological parameters that are crucial for optimizing lipoplex-mediated gene transfer.

Methods: A novel series of dicationic lipids (DCL) based on L-glutamic acid, a group of original lipopeptides with two quarternary ammonium polar heads were synthesized with different analogues, varying in the lenght of spacer arm between the two polar heads. The liposomes, their complexes with DNA (pEGFP-N1), and samples for AFM measurements were prepared as described elsewhere [3]. The potential of liposomes, prepared from the DCL, for delivering functional (reporter) genes into eukaryotic cells was examined by monitoring gene expression in different types of established cell lines, e.g. SKOV-3, 293 and MCF-7 [4].

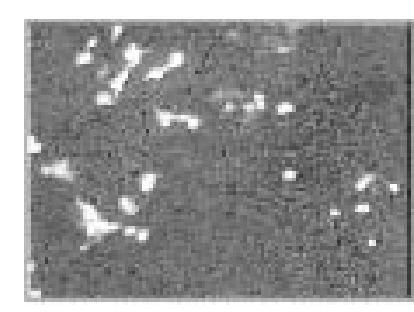

a)

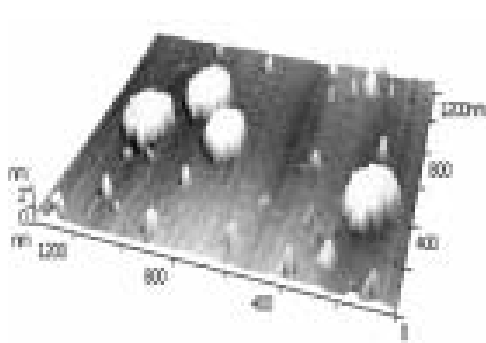

b)

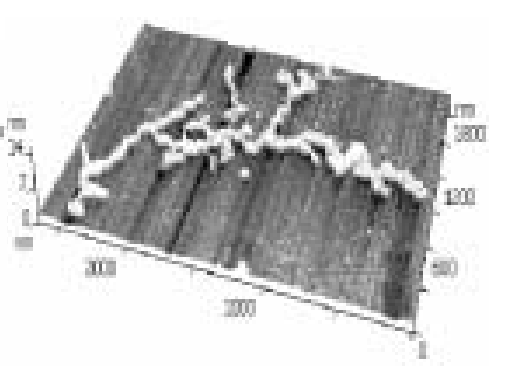

c)

Fig. 1. a) Transfection of human kidney transformed cell line 293 with pDNA-DCL complexes: transfected cells emit green fluorescence; b) AFM image for pDNA-DCL complexes taken after adhesion to the mica surface, shown are individual distinct toroids obtained with DCL with short spacer; c) Aggregation of toroids and rods obtained with DCL with long spacer.

Results and discussions: We have examined some of the biophysical characteristics of the interaction of DNA with several different cationic lipid analogues, varying in the length of the spacer arm between the 2 polar heads of lipid chain groups. The cationic lipids varied in their ability to mediate transfection, as it was found that lipoplexes composed from DCL with short spacers showed high transfection efficiency compared to those with long spacers (see Fig. 1(a)).

\footnotetext{
${ }^{*}$ Fax: +7 095 9328820; E-mail: ashraf_elkady@hotmail.com.
} 
The structural and morphological peculiarities of these lipoplexes were characterized using AFM. Interestingly, differences were observed in the aggregation behavior of the complexes structural units: While short spacer liposomes, exhibiting high transfection activity, form distinct compact condensed particles in toroid form with average diameter $\sim 120 \mathrm{~nm}$, which are stable against aggregation (Fig. 1(b)); the formation of multimolecular aggregates $(\sim 3 \mu \mathrm{m})$ of toroid and rod units has been noted for lipids with long spacers.

The size of the latter complexes exceeds that of plasmid and may acquire diameters in the order of microns. It is more likely that this fraction will be exluded from involvement in cellular transfection, as such a size will prevent cellular uptake [5].

Concluding remarks: The stability of the unit DNA-CL complex is an important consideration in the design or selection of cationic lipid for self-assembling DNA delivery systems. It is shown, that liposomes from DCL with short spacer form morphologically more compact particles of the toroidal form $(\sim 120 \mathrm{~nm})$ which are stable in solution (showed minimal aggregation of their DNA complexes) and effectively transfer functional genes to cultured cellular lines, while complexes formed from long spacer liposomes form the big, noncompact amorphous aggregates which are unnstable and do not transfer genes in cultured cells.

\section{References}

[1] P. Belmont, A. Aissaoui, M. Hauchecorne, N. Oudrhiri, L. Petit, J.P. Vigneron J.M. Lehn and P. Lehn, J Gene Med 4 (2002), 517-526.

[2] D. Lasic, Liposomes in Gene Delivery, CRC Press, Boca Raton, 1997.

[3] A. Elkady, Y. Sebyakin, M. Gallyamov, A. Moskovtsev, G. Bischoff, R. Zhdanov and A. Khokhlov, in: Micro- and Nanostructures of Biological Systems, G. Bischoff and H.-J. Hien, eds, Shaker Verlag, Aachen, 2003.

[4] R.I. Zhdanov, E.V. Bogdanenko, A.A. Moskovtsev, O.V. Podobed and N. Duzgunes, Methods in Enzymology. Liposomes, (N. Duzgunes, ed.) 2003, in press.

[5] V. Oberle, U. Bakowsky, I.S. Zuhorn and D. Hoekstra, Biophysical Journal 79(3) (2000), 1447-1454.

\section{Structural and morphological peculiarities of DNA-Vitamin D complexes: A fluorescence and atomic force microscopy study}

A.S. Elkady, S. Abramchuk and A.R. Khokhlov

Physics Department, Moscow State University, Moscow 119992, Russia

Fax: +7 095932 8820; E-mail: ashraf_elkady@hotmail.com

Introduction: In its genomic action, vitamin D (VD) binds to a specific receptor which is a member of the steroid hormone receptor superfamily. The vitamin D receptor (VDR) forms heterodimers with retinoid X receptors (RXRs), and the dimer interacts then with its cognate binding site, termed vitamin $\mathrm{D}$ response element (VDRE), to affect the transcription of target genes. Recent studies have identified novel sequence motifs for VDREs as well as novel protein-protein interactions involving the VDR. Thus, the research on the control of gene transcription by VD reveals examples of molecular interplay between transcriptional regulatory pathways and provides new insight into the molecular mechanism of action of VD [1]. However, detailed studies concerning direct interaction between DNA molecules and VD group (VD2 and VD3) are still lacking. In the present study, the self-assembly of phage T4 and plasmid 

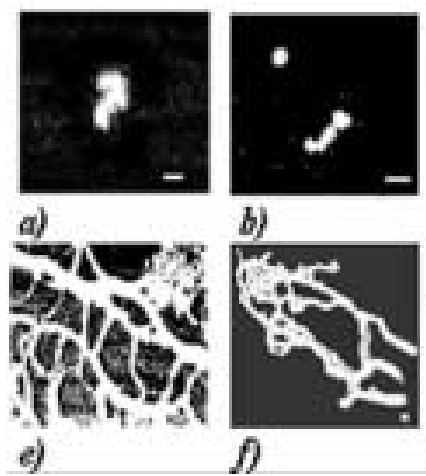

b)

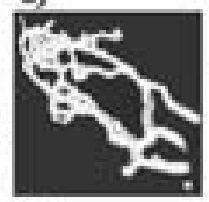

f)
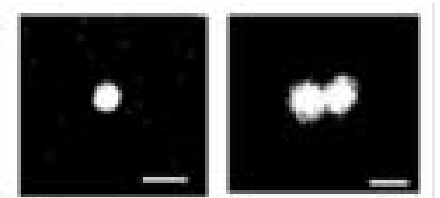

c)
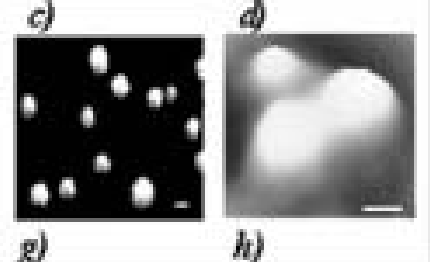

Fig. 1. FM (a-d) and AFM (e-h) images for DNA-VD complexes a) an elongated-coil; b) coexistence of coils and globules; c) globule; d) associated globules, note that due to a blurring effect, the apparent globule image is larger than the actual dimensions; e) formation of pDNA networks; f) aggregation of pDNA molecules around VD micelles; g) T-4 DNA globules; h) associated globules, see text for detailed description; the scale bars represent $1 \mu \mathrm{m}$ for FM images and $100 \mathrm{~nm}$ for AFM images.

DNA (pDNA) with VD group, was examined in diluted aqueous solutions, in the absence and presence of $\mathrm{Ca}^{2+}$ cations, using Fluorescence and Atomic Force Microscopy (FM and AFM, respectively).

Methods: Fluorescence images of T4 DNA molecules were observed using a Carl Zeiss microscope, Axiovert 135 TV, equipped with a highly sensitive CCD TV camera LCL-902 HS, and were recorded on H.D with a rate of 10 frames/s. Observations were carried out at the temperature $25^{\circ} \mathrm{C}$. Small droplets $(2 \mu \mathrm{l})$ of dilute solution of T4 DNA labelled by YOYO-1 in TE buffer solution in the presence/absence of VD were placed on glass cover-slip at the stage of the FM. AFM measurements were done following the method described elsewhere [2].

Results and discussions: To characterize the structural transition of DNA induced by vitamin D in a quantitative manner, a series of measurements was carried out by changing the $\mathrm{VD}$ and $\mathrm{Ca}^{+2}$ cations concentrations. A typical image of T4 DNA molecules, without any ligand, in a random coil state is given in Fig. 1(a)). In aqueous solutions in the absence of VD, individual DNA chains exhibited thermal fluctuation, i.e., intramolecular and translational Brownian motion in coil state. In contrast, after addition of $\mathrm{VD}(0.3 \mathrm{mg} / \mathrm{ml})$, the brownian motion for DNAs molecules was exteremely hindered (inhibited). With the addition of $\mathrm{Ca}^{+2}$ with different ratios, discrete conformational changes from an elongated coil into a collapsed globule took place in DNA molecules. A coexistence between coil and globule states was observed below $10 \mathrm{vol} \% \mathrm{Ca}^{+2}$ (Fig. 1(b)). At $10 \% \mathrm{Ca}^{+2}$, most of the DNAs observed were exhibiting the collapsed globule state (see Figs 1(c) and (g)), while a minor fraction showed the coiled state. Associations between multiple DNAs (aggregation) took place above $15 \% \mathrm{Ca}^{+2}$ (see Figs $1(\mathrm{~d})$ and (h)). At higher $\mathrm{Ca}^{+2}$ concentrations, we can hardly see any fluorescence from DNA molecules, which could be due to DNA full coverage by VD layers.

High resolution AFM images revealed a variety of structures for DNA- $\mathrm{Ca}^{2+}$-VD complexes. While at low VD2 concentrations, beads-on-strings strucure with the vitamin micelles attached to linearized pDNA was the predominant structure, formation of nanocylinderical networks (pDNA coated fibres) were obtained at rather high vitamin concentrations (Fig. 1(e)). Formation of loops around VD micelles and subsequent flower like aggregates was monitored as an early stage in condensate formation (see Fig. 1(f)). Surprisingly, incubation of T-4 DNA with VD3 (without any $\mathrm{Ca}^{2+}$ ) for 24 hours at $4{ }^{\circ} \mathrm{C}$, gave rise to formation of distinct globules, which associate (aggregate) in the presence of $\mathrm{Ca}^{2+}$ (see Figs $1(\mathrm{~g}$ ) and (h) respectively). 
In conclusion, we described a simple method for preparing DNA-VD complex in the existence of divalent cations. The DNA-Ca ${ }^{2+}-\mathrm{VD}$ complex structure ranged from amorphous aggregates; beadson-strings to compact single and multiple globules $(0.1-1 \mu \mathrm{m})$, depending on vitamin nature, $\mathrm{Ca}^{2+}$ concentration and incubation time. A nucleation mechanism and flower-like aggregates are proposed as an initial state for DNA-Ca ${ }^{2+}-$ VD complex formation. Finally, it's worth mentioning that the present study could have its fundamental and practical implications. Whereas from the fundamental viewpoint, it may highlight new mechanism of regulation of the genomic action of VD; it could also practically used as a novel approach for vitamin mediated targeted gene delivery.

\section{References}

[1] S.-A. Rene et al., Frontiers in Bioscience 1 (1 August 1996), d177-188.

[2] A.S. Elkady et al., Proceedings of the international conference on Scanning Probe Microscopy SPM-2002, Nizhny Novgorod, Russia, March 3-6, 2002, pp. 181-183.

\section{Fibrin as a carrier for chondrocytes and release systems for bioactive molecules in cartilage engineering}

D. Eyrich, A. Maschke, M. Wenzel, R. Staudenmaier, T. Blunk and A. Göpferich Department of Pharmaceutical Technology, University of Regensburg, 93040 Regensburg, Germany Tel.: +49 941943 5042; Fax: +49 941943 4807; E-mail: daniela.eyrich@chemie.uni-regensburg.de

\section{Introduction}

Hydrogels are a promising scaffold material with various functions in the field of tissue engineering. Our work is focused on using a fibrin gel as a three-dimensional carrier for chondrocytes and as a release system for bioactive molecules in cartilage engineering for total ear reconstruction. Fibrin is a well-investigated medical device and has been used for over 20 years in clinical and laboratory medicine [3]. It is generally considered to be biodegradable and biocompatible. However, using a commonly employed commercially available fibrin gel preparation kit, the fibrin hydrogel appeared unstable, shrunk considerably during the first few days following preparation, and completely dissolved after 3 weeks. For this reason, we investigated preparation parameters determining gel stability.

Within these gels an insulin-sensitive chondrocyte culture was established for evaluating controlled release of bioactive molecules from microparticles, using insulin as a potent cartilage-effective model drug [2]. Insulin is a polypeptide, is homologous to parts of the IGF-I molecule, and binds to the IGF receptor, though with 100-fold lower affinity than IGF-I. Within this study, we tested the effect of insulin released from lipid microparticles on chondrocytes grown in a fibrin gel.

\section{Methods}

Fibrin gel development: Fibrin gels principally consist of fibrinogen that is cross-linked by thrombin in $\mathrm{CaCl}_{2}$-buffer after a few seconds [1]. We modified the concentration of fibrinogen, $\mathrm{CaCl}_{2}$, and the $\mathrm{pH}$ and tested the appearance and the stability of the resultant gels in culture medium for 3 weeks. 
Table 1

Gel appearance and stability in culture medium; effect of fibrinogen concentration

\begin{tabular}{|c|c|c|c|c|}
\hline \multirow[t]{2}{*}{ Components } & \multirow{2}{*}{$\begin{array}{l}\text { Variation } \\
\text { Fibrinogen }\end{array}$} & \multicolumn{3}{|c|}{ Appearance } \\
\hline & & after gelling & after 1 week & after 3 weeks \\
\hline $\begin{array}{l}\text { commercial fibrin glue } \\
\text { Sigma Fibrinogen and } \\
\text { Thrombin } 5 \mathrm{U} / \mathrm{ml} \text {, } \\
40 \mathrm{mM} \mathrm{CaCl}_{2}, \mathrm{pH} 7.0\end{array}$ & $\begin{array}{l}- \\
12.5 \mathrm{mg} / \mathrm{ml} \\
25 \mathrm{mg} / \mathrm{ml} \\
50 \mathrm{mg} / \mathrm{ml} \\
100 \mathrm{mg} / \mathrm{ml}\end{array}$ & $\begin{array}{l}\text { turbid, some contraction } \\
\text { turbid, some contraction } \\
\text { turbid, some contraction } \\
\text { transparent } \\
\text { transparent }\end{array}$ & $\begin{array}{l}\text { turbid, some dissolution } \\
\text { turbid, some dissolution } \\
\text { turbid, some dissolution } \\
\text { stable, transparent } \\
\text { stable, transparent }\end{array}$ & $\begin{array}{l}\text { dissolved } \\
\text { dissolved } \\
\text { almost completely dissolved } \\
\text { stable, transparent } \\
\text { stable, transparent }\end{array}$ \\
\hline
\end{tabular}

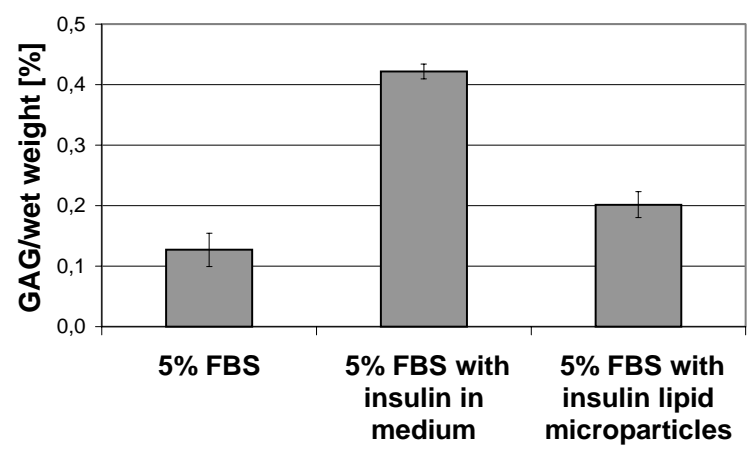

Fig. 1. Effects of insulin in medium and insulin-loaded lipid microparticles on GAG fraction of gels cultured for 5 weeks.

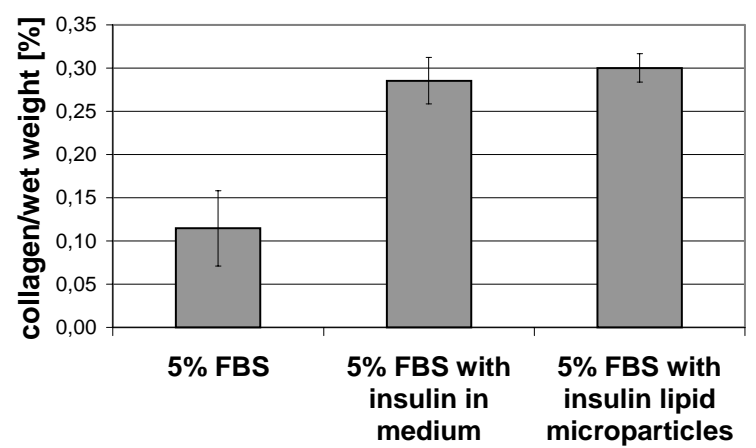

Fig. 2. Effects of insulin in medium and insulin-loaded lipid microparticles on collagen fraction of gels cultured for 5 weeks.

Cell culture: Freshly isolated bovine femoral chondrocytes were resuspended in a fibrinogen solution; subsequently gels were prepared in a $5 \mathrm{~mm}$ diameter glass ring according to the results obtained in the fibrin gel development $\left(1.5 \times 10^{6}\right.$ cells per $0.5 \mathrm{ml}$ gel $)$. The fibrin was allowed to gel for 1 hour at $37^{\circ} \mathrm{C}$ before removing the glass ring and covering the gel with $4 \mathrm{ml}$ culture medium. Medium was exchanged 3 times per week. For control cultures medium containing 5\% FBS was used, one experimental group received medium containing 5\% FBS and $2.5 \mu \mathrm{g} / \mathrm{ml}$ insulin. In the third group lipid microparticles loaded with insulin for controlled release were introduced into the gel together with the cell suspension before gelling, medium contained only 5\% FBS. The constructs were cultured in 12-well-plates and harvested after 2 and 5 weeks, respectively. 


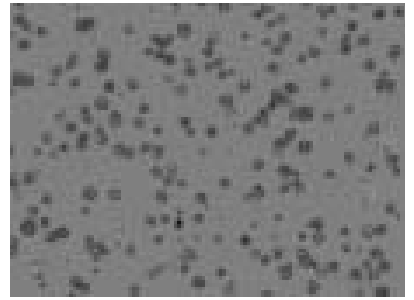

(A) control, 5\% FBS

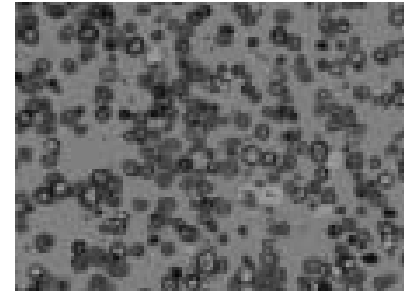

(B) $5 \% \mathrm{FBS}+$ insulin in medium

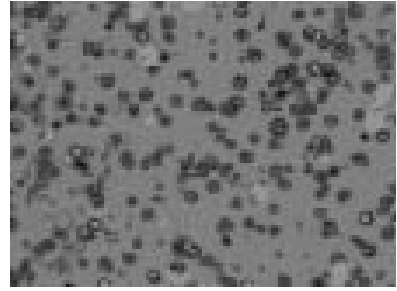

(C) $5 \%$ FBS + insulin-loaded lipid microparticles

Fig. 3. Histological cross-sections of 5-week gels grown in culture medium with (A) $5 \%$ FBS, (B) $5 \%$ FBS with $2.5 \mu \mathrm{g} / \mathrm{ml}$ insulin in medium, and (C) $5 \%$ FBS with insulin-loaded lipid microparticles. Sections were stained red for glycosaminoglycans (GAG) with safranin-O (x 100).

\section{Results}

Variation of fibrin concentration resulted in gels of distinctly different appearance (Table 1). Only a gel with a fibrin concentration higher than $50 \mathrm{mg} / \mathrm{ml}$ was at least stable for three weeks. With regard to $\mathrm{CaCl}_{2}$ and $\mathrm{pH}$, only a concentration higher than $40 \mathrm{mM} \mathrm{CaCl}_{2}$ and a pH between 6.8 and 9, respectively, result in stable gels (data not shown). In general, all gels that were turbid after preparation shrunk during the first few days and large parts dissolved within three weeks, i.e., an inappropriate period of time for long-term cell culture. In contrast, all transparent gels appeared stable and maintained their shape during the experiment. Based on the results from gel development, for cell culture experiments a preparation of $100 \mathrm{mg} / \mathrm{ml}$ fibrinogen dissolved in $10000 \mathrm{KIE} / \mathrm{ml}$ aprotinin solution at $37^{\circ} \mathrm{C}$ mixed with the same volume of thrombin $5 \mathrm{U} / \mathrm{ml}$ in $40 \mathrm{mM} \mathrm{CaCl}_{2}$-buffer was used. These gels have been stable in cell culture for 9 months up to now.

Primary chondrocytes in the fibrin gel cultured in medium with 5\% FBS (control group) maintained a round shape throughout the study and produced extracellular matrix containing GAG and collagen (Figs 1 and 2). Addition of insulin in the medium resulted in a distinct increase of GAG and collagen fraction in the gel (Figs 1 and 2). The GAG fraction of gels cultured in the presence of insulin-loaded lipid microparticles was slightly higher than that of control gels, but did not reach the amount produced in the presence of insulin in medium. However, as compared to the control, collagen fractions were increased by insulin-loaded particles to the same degree as by insulin exogenously added to the medium. (The generally low values for GAG and collagen fraction are attributed to the relatively low cell density within the gels; higher values are expected with increased cell/volume ratio.)

The different GAG fractions were also reflected in histological sections of the fibrin-cell constructs, stained red with safranin-O (Fig. 3). The cells were encircled by an area containing extracellular matrix. Compared to controls (Fig. 3(A)), these regions were larger and more heavily stained in the constructs with insulin in the medium (Fig. 3(B)) as well as with insulin in lipid microparticles (Fig. 3(C)). Cells with extracellular matrix were homogeneously distributed throughout the whole construct for all groups.

\section{Conclusions}

For the use of fibrin as a scaffold material, in many tissue engineering applications long-term stability is crucial. By varying preparation parameters, we produced a three-dimensional fibrin gel that has been stable in cell culture for 9 months up to the present and allows for suspension of chondrocytes, thereby maintaining their round morphology and phenotypic expression of glycosaminoglycan and 
collagen. Within these gels we established a chondrocyte culture that is sensitive to insulin as a model for bioactive molecules. Insulin released from lipid microparticles was demonstrated to improve chondrocyte extracellular matrix production. Future studies will further explore the effects of different protein loading and release kinetics from microparticles. The fibrin gels are intended to be specifically investigated in combination with polymeric scaffolds for the use in total ear reconstruction.

\title{
References
}

[1] B. Blombäck, Fibrinogen and fibrin - proteins with complex roles in hemostasis and thrombosis, Thrombosis Research 83(1) (1996), 1-75.

[2] K. Kellner, M.B. Schulz, A. Göpferich and T. Blunk, Insulin in tissue engineering of cartilage: a potential model system for growth factor application, Journal of Drug Targeting 9 (2001), 439-448.

[3] F.H. Silver, M.-C. Wand and G.D. Pins, Preparation and use of fibrin glue in surgery - review, Biomaterials 16 (1995), 891-903.

\section{Extended lifespan of porcine hepatocytes by transfection with the catalytic subunit of telomerase}

\author{
Wolfgang Fiedler ${ }^{\mathrm{a}, \mathrm{b}}$, D. Reinicke ${ }^{\mathrm{a}, \mathrm{b}}$, H. Aurich ${ }^{\mathrm{a}}$, B. Christ ${ }^{\mathrm{a}}$, W. Fleig ${ }^{\mathrm{a}}$ and W.G. Ballhausen ${ }^{\mathrm{a}, \mathrm{b}}$ \\ ${ }^{\mathrm{a}}$ Klinik und Poliklinik für Innere Medizin I, ${ }^{\mathrm{b}}$ Sektion Molekulare Gastroenterologische Onkologie, \\ Martin-Luther-Universität Halle-Wittenberg, Halle, Germany \\ Tel./Fax: +49345 5522944 3; E-mail: wolfgang.fiedler@medizin.uni-halle.de
}

Introduction: As in vitro maintenance of primary hepatocytes is difficult the availability of liver cells is limited. Improvement of the expansion of hepatocytes in cell culture would provide a new source of liver cells for several downstream applications. It has been shown for some human somatic cells that the reconstitution of telomerase only resulted in infinite proliferation without oncogenic transformation [2]. Thus, as an initial model, we tried to stimulate the proliferation of porcine hepatocytes by ectopic expression of the gene for the human catalytic subunit of telomerase reverse transcriptase (hTERT).

Materials and methods: For isolation of hepatocytes liver lobes of young male pigs were perfused with L15 Leiboviz medium for removing blood after total hepatectomy. The lobes were first washed with calcium free Krebs Ringer buffer followed by perfusion with Krebs Ringer-HEPES buffer containing calcium and $0.025 \%$ collagenase type 1 . Hepatocytes were separated from the disintegrated lobe by gentle agitation. The resulting cell suspension was washed by centrifugation three times at $50 \mathrm{~g}$ at $4{ }^{\circ} \mathrm{C}$. The final cell pellet was resuspended in ME medium to a density of $1 \times 10^{7}$ cells per ml. $3 \times 10^{5}$ cells per ml were plated onto $75 \mathrm{~cm}^{2}$ tissue culture dishes coated with collagen and then cultured in particular supplemented WE medium. In each experiment $2.25 \times 10^{6}$ cells were transfected with hTERT cloned in an eukaryotic expression plasmid using Metafectene (Biontex) as transfection solution. TRAP assays were performed as described previously by Fiedler et al. [1]. The conditions of RT-PCR analysis are available on demand from the author.

Results: In two of four independent approaches the lifespan of porcine hepatocytes could be extended by ectopic expression of hTERT. These hTERT transfected cells still proliferate after 8 months under specific culturing selection, whereas the non transfected control cells stopped proliferation and died in the meanwhile. Investigation of the telomerase activity by TRAP assay revealed that telomerase is active only in the transfected cells. Furthermore, expression of hTERT in these telomerase active cells 
was confirmed by RT-PCR. The hepatocytic character, the oncogenic potential, the karyotype and the telomere lengths of the cells are currently under investigation.

Discussion: The two cell lines established by ectopic expression of hTERT in primary porcine hepatocytes show indefinite proliferation capacity and seem to be immortal so far. Thus, in these cells limited in vitro lifespan of primary cultured hepatocytes was obviously overcome by telomerization. The generation of immortalized porcine hepatocytes by ectopic expression of telomerase without oncogenic transformation will be useful as an ex vivo animal model for hepatic gene therapy and further in vitro studies, but it may also be a first step to the development of human bioartifical liver devices.

\section{References}

[1] Fiedler et al., Kidney Int 56 (1999), 1286-1288.

[2] Oulton and Harrington, Curr Opin Oncol 12 (2000), 74-81. 